\title{
Die Zukunft des europäischen Verfassungstopos und Primärrechts nach der deutschen Ratspräsidentschaft
}

\author{
Peter-Christian Müller-Graff*
}

Die Zukunft des europäischen Verfassungstopos und Primärrechts nach der deutschen Ratspräsidentschaft im unmittelbaren Nachgang zum Europäischen Rat vom 21./22. Juni 2007 von einem Europarechtler beleuchten zu lassen, ${ }^{1}$ unterstreicht, dass die europäische Integration nach politischer Reflexionsphase, versierter Taktik und negotionalem Pulverdampf in die ruhigeren Fahrwasser nüchterner juristischer Feinmechanik geraten ist. Tatsächlich liegt seit dem Morgen des 23. Juni 2007 dank des Geschicks der Ratspräsidentschaft ein ungewöhnlich ausgefeiltes Mandat für die nächste Regierungskonferenz zur Ausarbeitung eines Reformvertrages zur Änderung der bestehenden Verträge vor, ${ }^{2}$ das bereits einen hohen juristischen Präzisionsgrad aufweist. Es ist in dieser Hinsicht mithin deutlich unterschieden von jenem fragenreichen Mandat von Laeken für den Europäischen Konvent, ${ }^{3}$ das dieser zur Ausarbeitung eines Vertrages für eine Verfassung für Europa nutzte. ${ }^{4}$ Für die Zukunft des europäischen Primärrechts und Verfassungstopos ist der Blick nachfolgend in einem Dreischritt zu richten: erstens und schwerpunktmäßig auf den Mandatsinhalt, sodann zweitens auf die Mandatsausfüllung und drittens auf den europäischen Verfassungstopos.

\section{Mand atsinhalt}

Wendet man sich zunächst dem 16-seitigen Mandatsinhalt in Anlage I der Schlussfolgerungen des Vorsitzes zu, so werden rasch drei Kennzeichen deutlich: er ist erstens inhaltlich grundsätzlich den Neuerungen des Verfassungsvertrages verpflichtet; zweitens in seinen Abweichungen entweder auf Vermeidung vermuteter öffentlicher Besorgnisse gerichtet oder neuen politischen Konstellationen beziehungsweise Einsichten geschuldet; und drittens normativ sehr kleinteilig verfasst.

\section{Inhaltliche Kennzeichen}

Herausragend ist hierbei die Orientierung am Verfassungsvertrag. Ziffer 1 des Mandats setzt den Ton: „Mit dem Reformvertrag sollen in die bestehenden Verträge, die weiterhin in Kraft bleiben, die auf die RK 2004 zurückgehenden Neuerungen in der nachstehend im Einzelnen beschriebenen Weise eingearbeitet werden. “Ziffer 4 wiederholt dies mit den Worten: „Was die inhaltlichen Änderungen an den bestehenden Verträgen anbelangt, so werden die auf die RK 2004 zurückgehenden Neuerungen so, wie es in diesem Mandat angegeben ist, in den EUV und den Vertrag über die Arbeitsweise der Union eingearbeitet. Änderungen an

Prof. Dr. Dr. h.c. Peter-Christian Müller-Graff, Universität Heidelberg.

1 Schriftliche Fassung des Vortrags, den der Verfasser am 27.6.2007 im Nachgang zum Europäischen Rat vom 21./22.6.2007 im Rahmen des „Forum Constitutionis Europae“ des Walter Hallstein-Instituts für Europäisches Verfassungsrecht an der Humboldt-Universität Berlin gehalten hat.

2 Vgl. Rat der Europäischen Union: Schlussfolgerungen des Vorsitzes, 23. Juni 2007, Dok. 11177/07, Anlage I, S.15ff.

3 Vgl. dazu Sebastian Barnutz/Martin Große Hüttemann: Die Verfassungsdebatte nach Laeken: der Konvent als neue und bessere Methode für Reformen in der EU?, in: integration 2/2002, S. 157-163.

4 Vgl. dazu die umfassende Bewertung in der Ausgabe der integration: Der Verfassungsentwurf des Europäischen Konvents, integration 4/2003, S. 283-575. 
diesen Neuerungen, die sich aufgrund der in den vergangenen sechs Monaten mit den Mitgliedstaaten geführten Konsultationen ergeben, sind nachstehend eindeutig angegeben." Und Ziffer 18 besagt zur Änderung des EG-Vertrages: „Die auf der RK 2004 vereinbarten Neuerungen werden durch spezifische Änderungen in der üblichen Weise in den Vertrag eingefügt.“ Mithin: Ohne den Verfassungsvertrag namentlich zu nennen, ist der Bezug auf die Regierungskonferenz (RK) 2004 nichts anderes als der verschleierte Bezug auf die Neuerungen im Verfassungsvertrag. Sie sind das Rückgrat der angepeilten Reformen.

Sie betreffen laut Mandat (unbeschadet einer längeren Liste von Änderungen an den Ergebnissen der RK 20045) speziell für den derzeitigen EG-Vertrag „die Zuständigkeitsarten und -bereiche, den Anwendungsbereich der Beschlussfassung mit qualifizierter Mehrheit und der Beschlussfassung im Mitentscheidungsverfahren, die Unterscheidung zwischen Gesetzgebungsakten und Rechtsakten ohne Gesetzescharakter, unter anderem Bestimmungen über den Raum der Freiheit, der Sicherheit und des Rechts, die Solidaritätsklausel, die Verbesserungen hinsichtlich der Steuerung des Euro, horizontale Bestimmungen wie die Sozialklausel, spezifische Bestimmungen wie zu öffentlichen Dienstleistungen, Raumfahrt, Energie, Katastrophenschutz, humanitärer Hilfe, öffentlicher Gesundheit, Sport und Tourismus, Regionen in äußerster Randlage, Verwaltungszusammenarbeit sowie Finanzbestimmungen. “6 Die Liste von Änderungen der Neuerungen des Verfassungsvertrages betreffen demgegenüber insbesondere die Semantik und Symbolik, Zuständigkeitsfragen, die Gemeinsame Außenund Sicherheitspolitik, die Rolle der nationalen Parlamente, die Behandlung der Grundrechte-Charta und die verstärkte Zusammenarbeit in der polizeilichen und strafjustiziellen Zusammenarbeit. ${ }^{7}$

Aus alledem ergibt sich: Der rote Faden der Orientierung am Verfassungsvertrag zieht sich durch das gesamte Mandat. Betrachtet man die wesentlichen Einzelpunkte des Mandats vergleichend aus Sicht von Aufbau und Themen des Verfassungsvertrages, ${ }^{8}$ lassen sie sich unterteilen in sieben Gruppen: Reformbegründung, Gesamtkonstruktion der Union, Grundsätze (Definition, Ziele, Grundrechte, Unionsbürgerschaft), Kompetenzen, Organe, Kompetenzausübung und Sonstiges.

\section{Begründung der Reformnotwendigkeit}

Die Orientierung am Verfassungsvertrag beginnt mit der Begründung der Notwendigkeit des Reformvertrages aus den drei Zielen der Erhöhung der Effizienz, der Erhöhung der demokratischen Legitimität und der Erhöhung der Kohärenz des auswärtigen Handelns der erweiterten Union.

Lediglich das Ziel der normativen Vereinfachung oder Transparenz - einer der vier Richtpunkte für die Entwicklung der Union in der 23. Erklärung von Nizza ${ }^{9}$ - wird nicht nur nicht genannt, sondern sogar mit den Worten verabschiedet: „Das Verfassungskonzept, das darin bestand, alle bestehenden Verträge aufzuheben und durch einen einheitlichen Text mit

\footnotetext{
Vgl. Ziff. 19 des Mandats.

Vgl. Ziff. 18 des Mandats.

Vgl. Ziff. 4 des Mandats.

Vgl. Konferenz der Vertreter der Regierungen der Mitgliedstaaten: RK 2003/2004 - Vorläufige konsolidierte Fassung des Vertrags über eine Verfassung für Europa, CIG 86/04, 25.06.2004 und Konferenz der Vertreter der Regierungen der Mitgliedstaaten: Vertrag über eine Verfassung für Europa, CIG 87/1/04, 25.10.2004; dazu Peter-Christian Müller-Graff: Strukturmerkmale des neuen Verfassungsvertrages für Europa, in: integration 3/ 2004, S. 186-201, mit weiteren Nachweisen.

9 Vgl. Anlage IV, Ziff. 5 (Stichwort: Vertragsvereinfachung); dazu Peter-Christian Müller-Graff: Der PostNizza-Prozess - Auf dem Weg zu einer neuen europäischen Verfassung?, in: integration 2/2001, S. 208-221, hier S. 208f, S. $213 \mathrm{ff}$.
} 
der Bezeichnung ,Verfassung ' zu ersetzen, wird aufgegeben. "10 Nüchtern ist festzuhalten: Damit erweist sich die große Kodifikationsleistung des Konvents, nämlich die systematisch durchdachte und dogmatisch kohärente Gesamtkodifikation des Primärrechts in einem durchlaufenden Text, jedenfalls im Jahre 2007, für die politische Praxis als nicht verwirklichbar. Das neue Jahrhundert kann mithin nicht mit einer neuen großen Kodifikation beginnen - anders als etwa das 20. Jahrhundert mit dem Bürgerlichen Gesetzbuch. Man mag emotionsfrei feststellen: Der politischen Gegenwart ist es nicht gegeben, normative Kathedralen zu errichten.

\section{Grundkonstruktion der Europäischen Union}

Davon abgesehen, spurt jedoch das Mandat den Reformvertrag auch in der herzustellenden Grundkonstruktion der europäischen Ebene weitestgehend auf die Neuerungen des Verfassungsvertrages ein. Dies kommt sprachlich kompliziert und verdeckt einher. Denn zunächst hat es in Ziffer 2 den Anschein, als würden Union und Gemeinschaft als jeweils eigene Organisationen fortgesetzt, wenn gesagt wird, der Reformvertrag werde zwei wesentliche Artikel enthalten: einen zur Änderung des EU-Vertrages und einen zur Änderung des EG-Vertrages. Indes verbirgt sich dahinter die Neukonstruktion, die bereits der Verfassungsvertrag (Artt. I-7, IV-438 VVE) im Auge hat: nämlich die Verschmelzung von Union und Gemeinschaft zu einer einzigen Organisation mit einheitlicher Rechtspersönlichkeit und einheitlichem Namen „Europäische Union"11 unter Ausklammerung der Europäischen Atomgemeinschaft. ${ }^{12}$

Folgerichtig sieht das Mandat - wie schon der Verfassungsvertrag - vor, dass die Gemeinschaft ihren Namen verliert, wiewohl freilich gerade dieser Name das historische Novum der Supranationalität besser konserviert hätte. Stimmig dazu wird der Begriff, Gemeinschaft ‘ wie im Verfassungsvertrag textlich durchgängig durch den Begriff ,Union ' ersetzt. ${ }^{13}$ Plausibel dazu erhält der EG-Vertrag den Namen des dritten Teil des Verfassungsvertrages (Art. III-115 VVE), nämlich ,Vertrag über die Arbeitsweise der Europäischen Union“14 (abgekürzt AEUV; ${ }^{15}$ nachfolgend kurz A-Vertrag - er bleibt der Erstgeborene). Er wird ausdrücklich und sinnfällig gleichrangig zum EU-Vertrag gestellt, ${ }^{16}$ sodass es auch nicht zu der Zweiteilung im Sinne der seinerzeitigen Dehaene-Dreier-Gruppe kommt. ${ }^{17}$ Reaktiv dazu und teils in Anlehnung an den Verfassungsvertrag wird der EU-Vertrag neu in sechs Titel untergliedert ${ }^{18}$ unter Wegfall der drei Titel zu den Europäischen Gemeinschaften und der dritten Säule sowie unter Aufnahme von Neuerungen des Verfassungsvertrages (im projektierten Aufbau mithin: 1. wie bisher: Allgemeines; 2. neu: das demokratische Leben; 3. überwiegend neu: Organe; 4. wie bisher: verstärkte Zusammenarbeit; 5. gemischt neu und wie bisher: Gemeinsame Außen- und Sicherheitspolitik, die als einzige substanzielle Politik im EU-Vertrag verbleibt, sowie Allgemeines zum auswärtigen Handeln; 6. wie bisher: Schlussbestimmungen). Insgesamt aufgenommen wird dadurch der Gedanke der Profilbildung des

10 Vgl. Ziff. 1 des Mandats.

11 Vgl. Müller-Graff: Strukturmerkmale des neuen Verfassungsvertrages für Europa, 2004, S. 195.

12 Vgl. Ziff. 2 des Mandats.

13 Vgl. Ziff. 2 des Mandats.

14 Vgl. Ziff. 2 des Mandats.

15 Vgl. Ziff. 7 des Mandats Fn. 2.

16 Vgl. Ziff. 19 lit.a des Mandats: Beide Verträge haben „,den gleichen rechtlichen Stellenwert.“

17 Vgl. Jean-Luc Dehaene/David Simon/Richard von Weizsäcker: Die institutionellen Auswirkungen der Erweiterung, Bericht an die Europäische Kommission vom 18. Oktober 1999, S. 12f.; zum Problem dieses Vorschlags vgl. Müller-Graff: Der Post-Nizza-Prozess, 2001, S. 215.

18 Vgl. Ziff. 7 des Mandats. 
ersten Teils des VVE, ohne allerdings dessen Spannweite und Kohärenz zu erreichen. Passfähig wird die Definition der Verträge, auf denen die Union beruht, auf EU-Vertrag und AVertrag begrenzt ${ }^{19}$ und damit im Vergleich zum derzeitigen Recht ${ }^{20}$ der Euratom-Vertrag herausgenommen. ${ }^{21}$ Wenigstens in ihm lebt dadurch der Begriff ,Gemeinschaft ' fort, aber auch der EAG-Vertrag wird an die auf der RK 2004 beschlossenen Änderungen angepasst. ${ }^{22}$ Konsequent schließlich ist konzipiert, dass die zur Rechtsfähigkeit emporgehobene Union zum Rechtsnachfolger der Gemeinschaft - wie im Verfassungsvertrag für die dort neu projektierte Union (Art. IV-438 VVE) - wird..$^{23}$

\section{Grundsätze}

Auch die Grundsätze, genauer insbesondere die Bestimmungen über Ziele, Werte, Grundfreiheiten, ferner über das Verhältnis von Union und Mitgliedstaaten und die Grundrechte orientieren sich an den Neuerungen im Verfassungsvertrag, weisen aber auch Änderungen dazu auf.

Die für einen Gesellschaftsvertrag, den EG-Vertrag und Unionsvertrag vertragstypologisch seit jeher darstellen, wenig logische Voranstellung der Werte vor die Ziele ${ }^{24}$ wird aus dem Verfassungsvertrag (Art. I-2 und I-3 VVE) ebenso übernommen wie dessen Formulierung der Werte. Der ausgefalteten Zielformulierung des Verfassungsvertrags ${ }^{25}$ wird weitestgehend gefolgt, weist aber doch mehrere, bereits im Detail vorformulierte bemerkenswerte Neuerungen auf.

Dazu zählt insbesondere, dass zum ersten operativen Hauptziel der Union der sogenannte Raum der Freiheit, der Sicherheit und des Rechts nunmehr ohne textliche Verbindung zum Binnenmarkt erhoben wird. Der Binnenmarkt, der derzeit als erster Hauptverwirklichungsweg der Ziele der Europäischen Gemeinschaft figuriert (Art. 2 EG-Vertrag), ${ }^{26}$ erscheint im Mandat in einem nachgestellten Absatz, wobei die Akzentuierung des Verfassungsvertrags „mit freiem und unverfälschtem Wettbewerb“ aufgegeben und der Hinweis auf ein System, das den Wettbewerb vor Verfälschungen schützt (derzeit Art. 3 lit.g EG-Vertrag), in nahezu anstößig zu nennender Weise in ein seltsam verdreht formuliertes Protokoll verbannt werden soll. ${ }^{27}$ Dies alles ist ein konzeptionell und symbolistisch wenig überzeugender Eingriff, der den sinnfälligen Aufgabenaufbau der Union verkennt, ${ }^{28}$ wohl auch die unionale Ambition

19 Vgl. Ziff. 2 des Mandats; siehe auch Anlage 1, Ziff. 2.

20 Vgl. Artt. 1 Abs. 3, 10 EUV.

21 Vgl. Ziff. 2 des Mandats.

22 Vgl. Ziff. 23 des Mandats.

23 Vgl. Ziff. 2; siehe auch Anlage 1 Ziff. 2.

24 Da die Werte des Art. I-2 VVE auch als Ziele des Art. I-3 Abs. 1 VVE figurieren, lässt sich Art. I-2 VVE insoweit als herausgehobene vorlaufende Zielbestimmung verstehen.

25 Vgl. Müller-Graff: Strukturmerkmale des neuen Verfassungsvertrages für Europa, 2004, S. 191ff.

26 Vgl. dazu Peter-Christian Müller-Graff: Verfassungsziele der EG/EU, in: Manfred Dauses (Hrsg.): Handbuch des EU-Wirtschaftsrechts, 2000, A I Rdz. 90ff., 99ff.

27 Vgl. Mandat Anl. 1 Ziff. 3 Fn. 16: ,Den Verträgen wird das folgende Protokoll beigefügt: ,Protokoll über den Binnenmarkt und den Wettbewerb. Die Hohen Vertragsparteien sind in der Erwägung, dass zu dem Binnenmarkt, wie er in Artikel 3 des Vertrags über die Europäische Union beschrieben wird, ein System gehört, das den Wettbewerb vor Verfälschungen schützt, übereingekommen, dass die Union zu diesem Zweck erforderlichenfalls nach den Vertragsbestimmungen, einschließlich des Artikels 308 des Vertrags über die Arbeitsweise der Europäischen Union, tätig wird. “" Irritierend ist die Relativierung ,erforderlichenfalls“ im Hinblick auf die primär- und sekundärrechtlichen Wettbewerbsregeln des derzeitigen EG-Vertrags. Zur Spannweite der unmittelbaren Anwendbarkeit der Wettbewerbsregeln vgl. Peter-Christian Müller-Graff: Kommentierung der Artt. III-161ff. VVE, in: Christoph Vedder/Wolff Heintschel von Heinegg (Hrsg.): Europäischer Verfassungsvertrag, Baden-Baden 2007, S. 453ff.

28 Vgl. zum Zusammenhang des Raums der Freiheit, der Sicherheit und des Rechts mit dem Binnenmarkt PeterChristian Müller-Graff: Der „Raum der Freiheit, der Sicherheit und des Rechts“ im neuen Verfassungsvertrag für Europa - Neuerungen und Notwendigkeit seiner primärrechtlichen Rekonstruktion, in: Charlotte Gaitanides/Stefan Kadelbach/Gil Carlos Rodriguez Iglesias (Hrsg.): Europa und seine Verfassung, Festschrift für Manfred Zuleeg, Baden-Baden 2005, S. 605ff. 
über das unionale Vermögen stellt ${ }^{29}$ und noch dadurch verschlimmert wird, dass anscheinend die Gewährleistung der unmittelbar anwendbaren transnationalen Marktgrundfreiheiten anders als im Verfassungsvertrag (Art. I-4 VVE) ${ }^{30}$ nicht auf dem Fuße der Zielbestimmung folgt. Insgesamt liegt darin eine bizarre Verschleierung des substanziellen Antriebs der funktionalen Integrationsverdichtung seit Anbeginn, vielleicht langfristig sogar eine semantisch vermittelte Schwächung. Was aber wäre die Integration denn ohne ihn - auch in Zukunft?

Es gibt aber auch Positives in der erneuten Änderung der Zielbestimmung. Anders als im Verfassungsvertrag wird nunmehr die Errichtung einer Wirtschafts- und Währungsunion systemstimmig in den Zielartikel eingestellt. Sie wird zwar in der Reihung unglücklich nach vielen anderen internen Einzelzielen loziert, aber immerhin wird der derzeit nach Art. 2 EGVertrag maßgebliche zweite Hauptverwirklichungsweg der Ziele der EG ${ }^{31}$ wieder offenbart.

Aus den Grundsätzen des Verfassungsvertrags wird auch die Bestimmung zu den Beziehungen zwischen Union und Mitgliedstaaten (Art. I-5 VVE) übernommen (Stichworte: Achtung von Gleichheit, nationaler Identität und grundlegenden Staatsfunktionen; Prinzip der loyalen Zusammenarbeit). Allerdings wird sie um zwei Aussagen ergänzt:

Zum einen wird zur Achtung der grundlegenden Staatsfunktionen hinzugefügt, dass insbesondere die nationale Sicherheit weiterhin in die alleinige Zuständigkeit der einzelnen Mitgliedstaaten fällt, und damit die neue primärrechtliche Kategorie der ausschließlichen Zuständigkeit der Mitgliedstaaten eingeführt. Zum anderen wird dem Beziehungsartikel nunmehr politisch fanfarenartig und normlogisch plausibel die Kompetenzregel vorangestellt, dass alle der Union nicht übertragenen (sprachnormativ besser wäre ,zugeordneten ') Zuständigkeiten gemäß dem Grundsatz der begrenzten Einzelermächtigung bei den Mitgliedstaaten verbleiben. ${ }^{32}$ Andererseits liegt auf der Mandatslinie des Wegduckens die Nichtübernahme des Vorrangartikels des Verfassungsvertrags (Art. I-6 VVE). Stattdessen wird auf eine bereits vorformulierte Erklärung der projektierten RK 2007 ausgewichen, die auf die einschlägige Vorrang-Rechtsprechung des EuGH verweist. ${ }^{33}$ Dies ist ein politisch vielleicht opportunes Vorgehen, wirkt allerdings verschleiernd.

Schließlich soll der Reformvertrag den Inhalt der Grundrechte-Charta in der Fassung von Teil 2 des Verfassungsvertrages (Art. II-61ff. VVE) durch Verweis primärrechtsverbindlich machen, ohne diese aber volltextlich in den EU-Vertrag zu inkorporieren. ${ }^{34}$ Dies lässt sich gelassen sehen. Angesichts der proportionensprengenden Länge, ${ }^{35}$ dem teils überschießen-

29 Der Raum der Freiheit, der Sicherheit und des Rechts fußt primärrechtlich auf Ermächtigungsgrundlagen, während der Binnenmarkt auf unmittelbar anwendbaren Grundfreiheiten beruht. Die Nutzung der Ermächtigungsgrundlagen ist vom politischen Willen abhängig und erfolgte bislang ganz unterschiedlich (vgl. z.B. zur Ausfüllung Peter-Christian Müller-Graff/Friedemann Kainer: Asyl-, Einwanderungs- und Visapolitik, in: Werner Weidenfeld/Wolfgang Wessels (Hrsg.): Jahrbuch der Europäischen Integration 2006, Baden-Baden 2007, S. 137-144 mit weiteren Nachweisen; siehe auch Peter-Christian Müller-Graff: Der Raum der Freiheit, der Sicherheit und des Rechts - Der primärrechtliche Rahmen, in: Peter-Christian Müller-Graff (Hrsg.): Der Raum der Freiheit, der Sicherheit und des Rechts, Baden-Baden 2005. Überdies lässt sich ,Sicherheit" nicht ohne Durchsetzungskräfte verwirklichen, über die die Union selbst gerade nicht verfügt. Sie ist entscheidend auf die Loyalität der Mitgliedstaaten angewiesen.

30 Dazu Müller-Graff: Strukturmerkmale des neuen Verfassungsvertrages für Europa, 2004, S. 192.

31 Dazu Müller-Graff: Verfassungsziele der EG/EU, 2000, Rdz. 114ff.

32 Vgl. Mandat Anl. 1 Ziff. 4; siehe ferner die vorgesehene Erklärung gemäß Ziff. 19 lit. b Fn. 10 a des Mandats.

33 Vgl. Ziff. 3 Fn. 1 des Mandats.

34 Vgl. Ziff. 9 des Mandats (,Querverweis‘ im Artikel über Grundrechte) und Ziff. 9 Fn. 3 des Mandats (,Daher wird der Text der Charta der Grundrechte nicht in den Verträgen enthalten sein“) sowie Anl. 1 Ziff. 6: „die Charta der Grundrechte hat dieslbe Rechtsverbindlichkeit wie die Verträge“.

35 Im VVE dessen Art. II-61 - Art. II-114. 
den Inhalt ${ }^{36}$ und den kategorialen Sonderheiten ${ }^{37}$ der Charta ist dies sogar vernünftig. Überdies klinkt sich Britannien hier aus der unmittelbaren Anwendbarkeit vor nationalen Gerichten bei der Überprüfung nationalen Handelns (Erstreckungsrechtsprechung) aus ${ }^{38}$ und verfestigt damit seinen Status am normativ äußeren Integrationsrand.

\section{Kompetenzen}

Hinsichtlich der Neuerungen zu den Kompetenzregeln ist zu unterscheiden zwischen den allgemeinen Kompetenzregeln und den spezifischen Befugnissen.

Hinsichtlich der transparenten allgemeinen Kompetenzregeln des Verfassungsvertrages zu Prinzipien, Arten und Bereichszuordnung (Art. I-11ff. VVE) sieht das Mandat auffälligerweise nicht vor, sie in den EU-Vertrag zu übernehmen (mit Ausnahme, wie schon erwähnt, des Prinzips der begrenzten Einzelermächtigung ${ }^{39}$ ). Dies ist normkonzeptionell nicht sehr glücklich. Denn ohne Klärung des Kompetenzfeldes fehlt den nachfolgenden Organregeln des EU-Vertrages das Bezugsfeld ihres legitimierten Tätigkeitsbereichs. Immerhin sollen die für den Verfassungsvertrag erarbeiteten Kompetenzregeln allerdings ganz oder teilweise (das bleibt offen) an den Anfang des A-Vertrages gesetzt werden. ${ }^{40}$

Neuerungen zu den spezifischen Kompetenzen und der Ausweitung der qualifizierten Mehrheitsentscheidung werden an passfähiger Stelle im A-Vertrag ${ }^{41}$ beziehungsweise hinsichtlich der Gemeinsamen Außen- und Sicherheitspolitik im EU-Vertrag einzuarbeiten sein. Letzteres bedeutet freilich, dass die Regeln zum auswärtigen Handeln der Union im Vergleich zum Verfassungsvertrag (Art. III-292ff. VVE) normlokativ wieder auseinandergerissen werden, ${ }^{42}$ während diejenigen zum Raum der Freiheit, der Sicherheit und des Rechts (Art. III-257ff. VVE) zusammengefasst bleiben und im A-Vertrag plaziert sind. ${ }^{43} \mathrm{Da}$ die Union, wie gezeigt, eine einheitliche Rechtspersönlichkeit erhält, werden allerdings erstmals europäische Verbandskompetenzen für die Gesamtheit des Raums der Freiheit, der Sicherheit und des Rechts und für die Gemeinsame Außen- und Sicherheitspolitik geschaffen, die der Kompetenzart nach aber möglicherweise unklar bleibt ${ }^{44}$ (im Verfassungsvertrag wohl als geteilte Zuständigkeit zu verstehen ${ }^{45}$ ).

\section{Organe}

Hinsichtlich der institutionellen Neuerungen im Verfassungsvertrag (Art. I-19ff., III330ff. VVE) ist folgerichtig zur Aufteilung in profilbildende Elemente und Funktionsele-

36 Stichwort: Zweifelhafte Passgenauigkeit zu den Zuständigkeiten der Union.

37 Stichworte: Verbindung von Rechten und Grundsätzen; Maßgeblichkeit einzelstaatlicher Rechtsvorschriften und Gepflogenheiten; vgl. Art. II-112 VVE.

38 Vgl. Mandat Anl. 1 Ziff. 5 Fn. 19.

39 Vgl. Mandat Anl. 1 Ziff. 4.

40 Vgl. Ziff. 19 lit. b und c des Mandats sowie Ziff. 19 lit. b Fn.10 (vorgesehene „Erklärung zur Abgrenzung der Zuständigkeiten“ sowie vorgesehenes Protokoll über Begrenzung der Wirkungen der Ausübung einer geteilten Zuständigkeit).

41 Vgl. Ziff. 18 des Mandats.

42 Lediglich die Allgemeinen Bestimmungen über das auswärtige Handeln der Union und die Bestimmungen über die GASP erscheinen im EU-Vertrag, wohingegen etwa die Gemeinsame Handelspolitik im A-Vertrag verbleibt.

43 Vgl. Ziff. 7 Fn. 2 des Mandats.

44 Vgl. die insoweit ausdeutbare Ziff. 15 des Mandats; siehe auch die projektierte Erklärung der RK 2007 in Ziff. 15 Fn. 6 des Mandats.

45 Vgl. dazu die Formulierung des Art. I-14 VVE, die Art. I-15 und I-16 VVE einzubeziehen scheint; vgl. dazu Peter-Christian Müller-Graff: Die primärrechtlichen Grundlagen der Außenbeziehungen der europäischen Union - eine Skizze, in: Peter-Christian Müller-Graff (Hrsg.): Die Rolle der erweiterten Europäischen Union in der Welt, Baden-Baden 2006, S. 11, S. 15f. 
mente eine geteilte Einarbeitung vorgesehen: ${ }^{46}$ einerseits in den A-Vertrag, andererseits in den vorgesehenen neuen dritten Titel des EU-Vertrages, der sowohl den Überblick über das institutionelle System und die wichtigsten Änderungen enthalten soll: darunter die Zusammensetzung des Europäischen Parlaments; die Organstellung des Europäischen Rates; dessen Präsidentenamt; die Neudefinition der qualifizierten Mehrheit im Rat als (in sich jeweils qualifizierte) doppelte Mehrheit ab 2014 (mit - wohl in ein Protokoll zu setzenden - Übergangsregeln bis zum 31. März 2017 und einer danach erleichterten Ioannina-Klausel) ${ }^{47}$ die reduzierte Kommissionsgröße; die Schaffung des neuen Amtes eines Hohen Vertreters der Union für Außen- und Sicherheitspolitik; und die Neuerungen für den Gerichtshof. Legitimatorisch widersprüchlich und bedenklich ist freilich die fehlende Synchronität des Inkrafttretens der projektierten Ausweitung der qualifizierten Mehrheitsentscheidung und der Neudefinition der qualifizierten Mehrheit.

\section{Kompetenzausübung}

Im Hinblick auf die in den Art. I-33ff. VVE neuen Bestimmungen zur Kompetenzausübung wird das im Verfassungsvertrag entwickelte System der Rechtsakte, systematisch plausibel, im Wesentlichen in und nach dem derzeitigen Art. 249 EG-Vertrag eingestellt, ${ }^{48}$ allerdings auf die Bezeichnungen ,Gesetz‘ und ,Rahmengesetz 'verzichtet. ${ }^{49}$ Gleichwohl wird gewissermaßen in hinterer Linie die Unterscheidung des Verfassungsvertrages zwischen Gesetzgebungsakten (im ordentlichen und besonderen Verfahren) und Rechtsakten ohne Gesetzescharakter (Art. I-33f. VVE) beibehalten. ${ }^{50}$ Die Neuerungen des Verfassungsvertrages zur verstärkten Zusammenarbeit (Art. I-44 VVE) werden übernommen, ${ }^{51}$ lediglich das Teilnahmequorum unter Vermeidung neuer mathematischer Herausforderungen von $1 / 3$ auf neun Mitgliedstaaten umgestellt. ${ }^{52}$ In den A-Vertrag plaziert werden Neuerungen zur verstärkten Zusammenarbeit im Bereich der justiziellen Zusammenarbeit in Strafsachen und der polizeilichen Zusammenarbeit. ${ }^{53}$

\section{Sonstiges}

Schließlich werden aus dem ersten Teil des Verfassungsvertrages an prominente Stelle des EU-Vertrages insbesondere die Grundsätze zum demokratischen Leben in der Union (Art. I-45ff. VVE: Demokratische Gleichheit, repräsentative und partizipative Demokratie) transferiert. ${ }^{54}$ Sie werden zusätzlich bereichert durch die nunmehr systematische vertragsförmige Einbeziehung der nationalen Parlamente zum Funktionieren der Union bei gleichzeitiger Stärkung deren Rechte im Frühwarnverfahren (unter entsprechender Änderung des einschlägigen Protokolls). Dies fördert die parlamentarische Komponente, die das Europäische Parlament in seinen Begrenzungen des geltenden Konzepts alleine nicht hinreichend zu erbringen vermag. Übernommen werden schließlich weitere Neuerungen des Verfassungsvertrages: der Sache nach der Nachbarschaftsartikel (Art. I-57 VVE), ${ }^{55}$ modifizierte Neuerungen im Beitrittsverfahren (Art. I-58 VVE) ${ }^{56}$ und die Austrittsbestimmung (Art. I-60

46 Vgl. Ziff. 12 des Mandats.

47 Vgl. Ziff. 13 des Mandats.

48 Vgl. Ziff. 19 lit. u und v des Mandats.

49 Vgl. Ziff. 3 und 19 lit.v des Mandats.

50 Vgl. Ziff. 19 lit.v des Mandats.

51 Vgl. Ziff. 14 des Mandats.

52 Vgl. Ziff. 14 des Mandats und Art. I-44 Abs. 2 S.1 VVE.

53 Vgl. Ziff. 19 lit.1 des Mandats.

54 Vgl. Ziff. 11 des Mandats (Bestimmungen über demokratische Grundsätze) sowie Anl. 1 Titel II.

55 Vgl. Mandat Anl. 1 Ziff. 6.

56 Vgl. Ziff. 16 des Mandats sowie Anl. 1 Titel VI. 
VVE). ${ }^{57}$ Aus Teil 4 des Verfassungsvertrages in den EU-Vertrag überführt wird schließlich das dreiteilige System der Änderungsverfahren ${ }^{58}$ (Art. IV-443ff. VVE): die beiden vereinfachten Verfahren und das ordentliche Verfahren (und damit auch die Konventsmethode), bereichert um die an sich selbstverständliche, aber nunmehr optisch ausdrückliche Möglichkeit, Unionszuständigkeiten auch zu verringern. ${ }^{59}$

Summa summarum ist damit der weitaus größte Teil der Neuerungen des Verfassungsvertrags Teil der präskriptiven Dimension des Mandats.

\section{Gründe für die Orientierung am Verfassungsvertrag}

Es lohnt nach diesem Schnelldurchlauf durch das Mandat, einen Augenblick bei der Frage zu verweilen, worin die maßgeblichen Gründe zu sehen sind, dass der Reformvertrag derart präzise und magnethaft auf den Verfassungsvertrag orientiert bleibt. Gewiss ist hier zuallererst das unübersehbare Geschick und der lange Atem der deutschen Ratspräsidentschaft zu nennen. Dazu: Chapeau.

Daneben aber gibt es mindestens drei Gruppen von Gründen für die Orientierungskraft des Verfassungsvertrages.

\section{Akkumuliertes politisches Gewicht des Verfassungsvertrages}

Naheliegend ist erstens das akkumulierte politische Gewicht des Verfassungsvertrages. Es ist ein Gewicht, akkumuliert erstens aus der aufwendigen Erarbeitung des Grundtextes durch den breit legitimierten Konvent; zweitens aus seinem Charakter als politischer Gesamtkompromiss auf der RK 2004; drittens aus seiner Unterzeichnung durch alle seinerzeitigen Mitgliedstaaten (wenn auch auffälligerweise unter der Monumental-Statue von Innozenz $\mathrm{X}$, einem Gegner des Westfälischen Friedens); akkumuliert schließlich viertens aus der Ratifikation beziehungsweise der politischen Absegnung des Verfassungsvertrages durch 2/3 der Signatarstaaten. Dies alles wiegt schwer und umso überraschender wurden zu Recht Absetzbewegungen von Regierungen empfunden, die den Vertrag unterzeichnet hatten, ohne seine Ratifikation betrieben zu haben. Dies ist als ein bemerkenswerter und in der Integrationsgeschichte neuartig irritierender Vorgang zu registrieren.

\section{Konstanz unionsinterner und unionsexterner Rahmendaten}

Die zweite Gruppe von Gründen liegt in der Konstanz der maßgeblichen unionsinternen und unionsexternen Rahmendaten. Sie werden augenscheinlich und zu Recht als seit 2004 unverändert wahrgenommen: zum einen die Ziele gemeinsamer Friedenssicherung, Prosperitätsförderung, Solidarität und Selbstbehauptung auf weltweiter Ebene; zum anderen die daraus folgenden, genannten Erfordernisse der Erhöhung von Legitimität, Effizienz und auBenpolitischer Kohärenz der erweiterten Union.

\section{Normative Kraft der Systemrationalität}

Dass aber die wesentlichen Neuerungen des Verfassungsvertrages sich - von einigen Änderungen abgesehen - nachgerade punktgenau und prinzipienfest gegen alle Anfechtungen am Wegesrand der letzten sechs Monate durchsetzen konnten, hat meines Erachtens einen dritten, überaus mächtigen Grund. Er scheint geeignet, wie eine unsichtbare Hand dauerhaft

57 Vgl. Ziff. 16 des Mandats.

58 Vgl. Ziff. 16 des Mandats.

59 Ebenda. 
durch wechselnde Ratspräsidentschaften, aber auch durch wechselnde politische Wetterlagen in den Mitgliedstaaten zu leiten, jedenfalls soweit diese an dem europäischen Gemeinschaftswerk voll und nicht nur teilweise teilnehmen wollen. Es ist die normative Kraft der Systemrationalität des seit 1952 eingeschlagenen Integrationspfads, die ihren Ausdruck in den Neuerungen des Verfassungsvertrages gefunden hat. ${ }^{60}$ Meine These lautet: das Gewicht der Systemrationalität ist so überragend, dass sich an ihr bei unveränderten Rahmenbedingungen langfristig letztlich alle konzeptionsfremden Ansätze und Missverständnisse brechen. Ich nenne insbesondere vier Strömungen, die wiederkehrend und gerade in den vergangenen zwei Jahren die innere Grammatik des normativen Kern-Acquis missverstehen, auf die Bewährungsprobe stellen und potenziell zum Scheitern verurteilt sind: der klassischvölkerrechtliche Ansatz, der betont freihändlerische Ansatz, der staatsanaloge Ansatz und der blockierende Ansatz.

Das klassisch-völkerrechtliche Missverständnis der Europäischen Gemeinschaft wurde bei diesem Europäischen Rat vor allem symbolisiert vom Vorschlag der Quadratwurzel ${ }^{61}$ für die Definition der qualifizierten Mehrheit. Da es mit dieser Definition aber nicht allein um klassische zwischenstaatliche Politik geht, sondern um eine sinnfällige Lösung der Legitimationserfordernisse für supranationale Entscheidungen, ${ }^{62}$ namentlich von Rechtsetzungsakten, konnte die Quadratwurzel keinen Beitrag leisten. Denn supranational wirkende Mehrheitsentscheidungen verlangen nicht nur die völkerrechtliche Legitimation durch die betroffenen Staaten nach dem westfälischen Grundsatz der Gleichwertigkeit jedes Staates. Sie erfordern zugleich auch die demokratische Legitimation durch die betroffenen Unionsbürger nach dem demokratischen Grundsatz der Gleichwertigkeit jedes Unionsbürgers. Weil (und solange) dies von der primärrechtlichen Konzeption des Europäischen Parlaments aus verschiedenen Gründen nicht hinreichend geleistet wird, muss die Verwirklichung dieses Legitimationsgedankens im Rat abgesichert werden. ${ }^{63}$ Da die Quadratwurzel von der Prämisse der nach Staaten gegliederten Bevölkerung und damit der klassischen völkerrechtlichen Einteilung ausging, konnte sie nicht die grenzüberschreitende Gleichwertigkeit der betroffenen Bürger erfassen. Sie verfehlt mithin die supranationale Dimension des transnationalen Gemeinwesens und wirkt ebenso wie das Nizza-System ein wenig wie ein voraufgeklärter Hokuspokus der Gewichtung staatlicher Bedeutung. Demgegenüber steht die doppelte Mehrheit als systemrationale Lösung der Legitimationsfrage für die supranationale Dimension des transnationalen Gemeinwesens. ${ }^{64}$

Die betont freihändlerisch-wettbewerbliche Perzeption der Europäischen Gemeinschaft wurde dieses Mal besonders akzentuiert von einem Ökonomen im Präsidentenamt eines Mitgliedstaats. ${ }^{65}$ Noch im März forderte einer seiner wichtigsten Berater im Prager Außenministerium eine Rückkehr vor Maastricht - vor allem im Interesse eines Wettbewerbs ohne Regulativa. ${ }^{66}$ Gedankliche Verwandtschaften bestehen insoweit zu Ansätzen einer Reihe

60 Vgl. zu diesem Gedanken der Systemrationalität Peter-Christian Müller-Graff: Systemrationalität in Kontinuität und Änderung des europäischen Verfassungsvertrags, in: integration 4/2003, S. 301-316; Peter-Christian Müller-Graff: Eine neue Verfassung für Europa - Kriterien der Europarechtswissenschaft, in: Klaus Beckmann/Jürgen Dieringer/Ulrich Hufeld (Hrsg.): Eine Verfassung für Europa, 2. Auflage, Tübingen 2005, S. $311 \mathrm{ff}$.

61 Vgl. Frankfurter Allgemeine Zeitung: Quadratwurzel-Behandlung für Europa, 14.06.2007.

62 Vgl. dazu Müller-Graff: Strukturmerkmale des neuen Verfassungsvertrages für Europa, 2004, S. 197f.

63 Ebenda, S. 196ff.

64 Ebenda, S. 196ff.

65 Vgl. dazu das Interview mit Präsident Václav Klaus: Die Frage der Stimmengewichtung ist nicht entscheidend, in: Frankfurter Allgemeine Zeitung, 20.6.2007, S. 6.

66 Im Rahmen einer öffentlichen Diskussion auf der Internationalen Konferenz „European Integration at the Crossroads“, 16./17.3.2007. 
deutscher Ökonomen. Verkannt wird dadurch aber allzu leicht, dass gerade die Verwirklichung des erwünschten dauerhaften Freiverkehrs von Produktionsfaktoren und Produkten im System unverfälschten Wettbewerbs einer supranationalen Angleichungskompetenz der verschiedenen mitgliedstaatlichen Regulierungen zugunsten von Schutzgütern wie Gesundheit und Umwelt bedarf. In diesem Rahmen und in diesen Grenzen ist mithin im Interesse der Errichtung und des Funktionierens des Binnenmarktes eine transnational wettbewerbseröffnende Regulierungskompetenz unverzichtbar. ${ }^{67}$

Das staatsanalogisierende Missverständnis der Union stand in der schiefen und verkürzten Wahrnehmung des Verfassungsvertrages als ,EU-Verfassung' im Raum. Es ist müßig, darüber zu spekulieren, ob die Staatsanalogien in Gestalt von Verfassungssemantik und Symbolen der maßgebliche Grund der referendalen Ablehnung in Frankreich und den Niederlanden waren. Dies erscheint eher zweifelhaft. Naheliegender ist zunächst, dass ein mit den Protokollen 500-seitiger juristischer Text der filigranen Feinabstimmung zwischen seinerzeit 25 Staaten schon per se nicht seriös referendumfähig sein dürfte. ${ }^{68}$ Unter dem Gesichtspunkt der Systemrationalität zu erinnern ist aber vor allem, dass die auffällig und forciert staatsanalogisierende Verpackung nicht den spezifischen Charakter des Verfassungsvertrages und dessen Systemlogik in seiner gesamten Komplexität wiedergibt. Denn natülich handelt es sich um einen Vertrag. ${ }^{69}$ Und bei gewichteter Betrachtung seines Inhalts handelt es sich auch ersichtlich um ein Normwerk auf dem inhaltlich sinngebenden Sockel einer transnationalen Wirtschaftsordnung mit dem Richtpunkt grenzüberschreitender Wirtschaftsinitiative und dessen regulativen Konsequenzen, wie es etwa Werner Mussler ${ }^{70} \operatorname{sehr}$ rasch und treffend pointierte. Nicht aber ging es um eine Selbstkonstituierung europäischer Staatlichkeit oder um die Schaffung einer Bundesrepublik Europa.

Konsequenz dieser Kraft der Systemrationalität ist aber schließlich auch die Überwindung blockierender Ansätze durch Differenzierung. ${ }^{71}$ Denn Mitgliedstaaten, die sich an systemrational angezeigten Entwicklungen nicht beteiligen wollen, werden deren Inkraftsetzung für diejenigen Mitgliedstaaten, die sie verwirklichen wollen, auf Dauer nicht aufhalten können. Der Vertrag von Prüm ist ein jüngeres positives Beispiel, ${ }^{72}$ die erneute Verweigerung Britanniens an den Integrationsschritten der ungeschmälerten Ingeltungsetzung der Grundrechte-Charta ${ }^{73}$ und der Vergemeinschaftung im Bereich der justiziellen Zusammenarbeit $^{74}$ ist der jüngste Fall.

\section{Mand a ts a u s füllung}

Wendet man nun den Blick auf die Frage nach der Mandatsausfüllung und insbesondere nach den hierbei bestehenden Gestaltungsspielräumen, so ist bereits deutlich geworden,

67 Sie besteht namentlich in Gestalt des Art. 95 EG-Vertrages.

68 Aus dänischer Erfahrung zum Problem von Europapolitik durch Referendum vgl. Hjalte Rasmussen: Europäische Integration durch Referenden? - Dänische Erfahrungen, in: Peter- Christian Müller-Graff (Hrsg.): Europäisches Integrationsrecht im Querschnitt (Heidelberger Schriften zum Wirtschaftsrecht und Europarecht Band 10), Baden-Baden 2003, S. 87ff.

69 Vgl. Art. IV-437ff. VVE; Peter-Christian Müller-Graff: Strukturmerkmale des neuen Verfassungsvertrages für Europa, 2004, S. 189ff.

70 Wirtschaftskorrespondent der Frankfurter Allgemeinen Zeitung in Brüssel.

71 Zur konzeptionellen Seite vgl. Peter-Christian Müller-Graff: „Differenzierte Integration“: Konzept mit sprengender oder unitarisierender Kraft für Europäische Union?, in: integration 2/2007, S. 129-139.

72 Christoph Gusy/Christoph S. Schewe: Polizeiliche und justizielle Zusammenarbeit, in: Werner Weidenfeld/ Wolfgang Wessels (Hrsg.): Jahrbuch der Europäischen Integration 2006, Baden-Baden 2007, S. 191-200, hier S. $197 \mathrm{ff}$.

73 Vgl. Mandat Anl. 1 Ziff. 5 Fn. 19.

74 Vgl. Ziff. 19 lit. 1 des Mandats. 
dass, obwohl das Mandat naturgemäß nicht rechtsverbindlich ist, diese, anders als bei dem in Laeken dem Konvent erteilten Auftrag, politisch relativ gering zu sein scheinen. Dessen ungeachtet ist indes höchste Aufmerksamkeit und Sorgfalt bei der Mandatsausfüllung durch die bevorstehende Regierungskonferenz (RK 2007) geboten. Die Ausgangslage ergibt sich aus drei Kennzeichen:

\section{Orientierungsgrundsatz Verfassungsvertrag}

Erstens folgt aus dem Mandat, wie gezeigt, der politische Grundsatz der Orientierung des Reformvertrags an den Neuerungen des Verfassungsvertrages.

\section{Vorwegdefinierte Neuerungsänderungen}

Zweitens enthält das Mandat das politische Prinzip der kleinteiligen Änderungsbegrenzung, mit anderen Worten das Prinzip vorwegdefinierter Änderungen an den Neuerungen des Verfassungsvertrages. Sie manifestieren sich zum einen im Verzicht auf die Semantik ,Verfassung', ,Europäisches Gesetz' und ,Vorrang', auf die ,Symbole der Union', auf die textliche Inkorporation der Grundrechte-Charta und auf die geschlossene Systematik des Verfassungsvertrages. ${ }^{75}$ Sie zeigen sich zum anderen in einer Reihe punktgenau formulierter inhaltlicher Abweichungen vom Verfassungsvertrag (so für den EG-Vertrag: Liste von 24 Punkten ${ }^{76}$ einschließlich, beispielsweise, der Bekämpfung des Klimawandels und der Förderung der Interkonnektion der Energienetze ${ }^{77}$ ) und präziser Formulierungsvorgaben für Änderungen der Verträge, für Protokolle und Erklärungen sowie in genauen Gliederungs- und Lokationsangaben für Einzelbestimmungen. ${ }^{78}$

\section{Politisch begrenzte Gestaltungsspielräume}

Drittens folgt aus diesem Rahmen ein Terrain politisch begrenzter Formulierungs-, Gliederungs- und Gestaltungsspielräume für die bevorstehende RK 2007. Soweit sie vorhanden sind und soweit Detailfragen nochmals aufgenommen werden müssen, steht die designierte neue Ratspräsidentschaft mit zügigem Zeitplan schon bereit. Allerdings ist trotz des gedrängten Zeitplans die genaue Fassung des Reformvertrags (,Grundlagenvertrages ') mit Sorgfalt und Weitsicht vorzunehmen. Denn die nunmehr projektierte Reform des Primärrechts mag die letzte für lange Zeit bleiben. Dabei mögen mehrere allgemeine Leitlinien hilfreich sein.

\section{Konsistenz.}

Erstens sind Formulierungen in strenger Konsistenz mit der inneren und sprachlichen Systemrationalität von EG-Vertrag (projektiert A-Vertrag) und EU-Vertrag vorzunehmen.

\section{Systematisierungsniveau}

Zweitens ist das Systematisierungsniveau des Verfassungsvertrages zu erhalten, soweit dies vom Mandat nicht gehindert wird.

75 Vgl. Ziff. 1, 3, 9 des Mandats.

76 Vgl. Ziff. 19 des Mandats.

77 Vgl. Ziff. 19 lit.p und q des Mandats.

78 Zu letzterem vgl. Mandat Anl. 2 B. 


\section{Verbesserungen}

Drittens sind präzise Optionen zu Verbesserungen des vom Mandat vorgezeichneten Systematisierungs- und Lokationsniveaus zu bedenken, ohne damit freilich unnötig schwerwiegende Verhandlungskomplikationen loszutreten. Freilich ist es auch einem als kurzzeitig veranschlagten Reformverfahren nicht untersagt, in Einzelformulierungen klüger als das Mandat zu sein. Beispielhaft fallen bereits nach erster Analyse einige Punkte im Grundsatzbereich ins Auge.

Die Formulierung des Zielartikels (projektierter neuer Art. 3 EU-Vertrag) sollte nicht eine konzeptionell und substanziell missverständliche Verselbstständigung des Raums der Freiheit, der Sicherheit und des Rechts enthalten, sondern wie in Art. 3 Abs. 2 VVE sprachlich mit dem Binnenmarkt verklammert werden. Die vom Mandat vorgesehene Abtrennung verkennt den inneren Zusammenhang und verdeckt den Integrationssockel. ${ }^{79}$

Der Text des Zielartikels (projektierter neuer Art. 3 EU-Vertrag) sollte auch die Formulierung des Art. 3 Abs. 2 VVE beibehalten, dass die Union ihren Bürgerinnen und Bürgern einen Binnenmarkt mit freiem und unverfälschten Wettbewerb bietet. Die Auslagerung der ausdrücklichen Nennung des Wettbewerbsprinzips innerhalb der Union in ein Protokoll ist angesichts dessen strukturprägender Bedeutung für die Entwicklung der Integration und den Aufbau und Inhalt des gesamten Integrationsrechts seit einem halben Jahrhundert bizarr. ${ }^{80}$ Die Auslagerung kann für den alltäglichen Integrationsprozess künftig in allfälligen Diskussionen um die Marktöffnung politisch sogar schadensstiftend wirken.

Die transnationalen Grundfreiheiten sind wie in Art. I-4 VVE an herausgehobener vorderer Stelle des EU-Vertrages zu plazieren. Denn es sind die Grundfreiheiten, die als treibende innere Kraft des Integrationsprozesses seit einem halben Jahrhundert den EuGH zu maßgeblichen Erkenntnissen der Besonderheiten des Europäischen Gemeinschaftsrecht veranlassten: die Verleihung subjektiver Rechte der Einzelnen durch das primäre Gemeinschaftsrecht, ${ }^{81}$ die unmittelbare ${ }^{82}$ und vorrangige ${ }^{83}$ Anwendbarkeit des Gemeinschaftsrechts durch mitgliedstaatliche Gerichte und Behörden, die Entstehung einer neuen Rechtsordnung. ${ }^{84}$ Als die transnationalen Marktgrundfreiheiten im ersten Themenentwurf des Konventspräsidiums nicht auftauchten, protestierte unter anderem der seinerzeitige EuGH-Präsident Rodrigez Iglesias auf dem FIDE-Kongress in London $2002^{85}$ zu Recht und mit Erfolg. Dieser Protest sei hier vorsorglich erneuert.

Transparenzpolitisch und systematisch zweifelhaft ist die vom Mandat vorgesehene Lokation der Kompetenzregeln (Art. I-11ff. VVE) in den EG-Vertrag, zumal die unionale Verbandskompetenz zur Gemeinsamen Außen- und Sicherheitspolitik im EU-Vertrag figurieren soll. Alternativ wäre an die Verlagerung dieser Verbandskompetenz in das System des AVertrages zu denken.

79 Vgl. zu diesem Sockel Müller-Graff: Verfassungsziele der EG/EU, 2000, 28ff.

80 Vgl. Mandat Anl. 1 Ziff. 3 Fn. 16; zur Bedeutung des Wettbewerbsprinzips Müller-Graff: Kommentierung der Artt. III-161ff. VVE, 2007, S. 453ff.

81 Vgl. EuGH, Slg. 1963, 1 (van Gend \& Loos).

82 Ebenda.

83 Vgl. EuGH, Slg.1964, 1141 (Costa/ENEL).

84 Vgl. EuGH, 1963, (van Gend \& Loos) und EuGH, 1964, (Costa/ENEL).

85 Vgl. auch Peter-Christian Müller-Graff: Die Kopfartikel des Verfassungsentwurfs für Europa - ein europarechtlicher Vergleichsblick, in: integration 2/2003, S. 111-129, hier S. 111, S. $119 \mathrm{ff}$. 


\section{Das Problem der Dissynchronität}

Als politisch kaum klug auf der RK 2007 thematisierbare Position dürfte sich das Problem der zeitlich früher als das Inkrafftreten des Systems der doppelten Mehrheit projektierten Ausweitung der qualifizierten Mehrheit darstellen. Diejenigen, die diese Ausweitung ohne Synchronisierung wollen, müssen freilich hoffen, dass das Bundesverfassungsgericht weiterhin an seiner wohlwollenden Rechtsprechung der ausreichenden demokratischen Legitimation qualifizierter Mehrheitsentscheidungen festhält. Art. 205 Abs. 4 EG-Vertrag kann hierfür übergangsweise eine argumentative Hilfe bieten.

\section{Zukunft des Verfassungstopos}

Was aber, dritte und letzte Frage, bleibt bei alledem vom europäischen Verfassungstopos?

\section{Mandat}

Das Mandat sagt in Ziffer 1, wie schon zitiert, lakonisch, dass das Verfassungskonzept, das darin bestand, alle bestehenden Verträge aufzuheben und durch einen einheitlichen Text mit der Bezeichnung, Verfassung' zu ersetzen, aufgegeben wird. Die Frage ist freilich, ob damit der europäische Verfassungstopos überhaupt erfasst wird und dadurch Institute für Europäisches Verfassungsrecht mit einem Federstrich zu rechtshistorischen Forschungsstellen transformiert wurden. Ziffer 3 legt nach: „Der EUV und der Vertrag über die Arbeitsweise der Union werden keinen Verfassungscharakter haben.“ Die Frage ist allerdings, ob eine derartige semantische Qualifikation überhaupt der Definitionshoheit von Staats- und Regierungschefs zur Disposition steht.

Gewiss, der Verfassungsbegriff scheint vom Mandat abgelegt und seine politischen Funktionen verloren zu haben: die semantische Funktion, um das profilbildend Grundlegende des Zusammenwirkens und den Maßstab für Einzelmaßnahmen hervorzuheben; die fokussierende Funktion, um die gemeinwesenartige Natur der Union zu betonen; die perspektivische Funktion, um das Dauerhafte einer identitätsstiftenden Schicksalsgemeinschaft zu bezeichnen. Plötzlich wirkt der Verfassungsvertrag als kühnes Experiment, das den Test der politischen Wirklichkeit ebensowenig bestanden hat wie vor mehr als einem halben Jahrhundert die Europäische Verteidigungsgemeinschaft und die Europäische Politische Gemeinschaft. Aber ist damit die Funktion des Verfassungstopos im europäischen Kontext wirklich erledigt?

\section{Inhaltspotenzial}

Kaum ein anderes Wort hat in den Debatten der letzten Jahre vergleichsweise ebenso breitflächig geschillert, angeregt und provoziert wie die Redeweise von der europäischen Verfassung: eine unterschiedlich genutzte façon de parler, die im günstigsten Fall vom jeweiligen Nutzer präzisiert wurde, oft diffus blieb und im ungünstigsten Fall widersprüchlich einherkam. Vieles schwang an Oberfläche und in Tiefenschichten des Diskurses mit: Schicksalsgemeinschaft, Identitätsstiftung, Höchstrangautorität, Volkssouveränität, Europäischer Demos. Zweifelsohne hat zu dieser Vielschichtigkeit der Verfassungsvertrag auch Vorschub geleistet: mit Textelementen als forcierter Einstieg in eine Grauzone zwischen Staatlichkeit und Nichtstaatlichkeit: so die linguale Hinleitung zu einem Verständnis der Union als staatsartige Gemeinschaft und Identitätsbestandteil der Unionsbürger (namentlich mittels der durchlaufenden Selbstbezeichnung des Textes bis Art. III-436 VVE als „Verfas- 
sung“ und der Einführung staatsanaloger Symbole, obwohl Teil 4 ab Art. IV-437 VVE dann übergangslos zur juristisch korrekten Selbstbezeichnung als „Vertrag“ wechselt); vielleicht auch die Heranführung zu einer bundesstaatsartigen Wahrnehmung der Union durch die föderationsanaloge Themensammlung im ersten Teil, auch wenn die juristische Grenze nicht übersprungen wurde; möglicherweise auch die Förderung der Perzeption einer europäischen Selbstkonstituierung durch den wiederholten Legitimationsrekurs auf die Unionsbürgerschaft (des Gesamttextes in Art. I-1 VVE und der Abgeordneten des Europäischen Parlaments in Art. I-20 Abs. 2 VVE) und auch durch die vereinfachten Vertragsänderungsverfahren (Art. IV-444f. VVE), obwohl auch hier die Schwelle eines Vertrages zwischen Staaten nicht überschritten wurde. ${ }^{86}$ Es ist hier nicht der Anlass, das hermeneutisch Verleitliche solcher Formulierungen und Vorverständnisse aufzuzeigen, wenn es um die Bewertung des Verfassungsvertrages geht.

Aber auffällig war und ist es aus rechtswissenschaftlicher Sicht nun doch, dass die inhaltliche Kernfunktion von Verfassungstexten, wie sie sich seit mehr als zwei Jahrhunderten entwickeln, öffentlich bestenfalls am Rande zur Sprache kam. Denn Verfassungstexte hat es nicht schon immer gegeben. Sie haben sich vielmehr als eine Antwortform auf eine grundlegende zeitlose Frage entwickelt: Wer darf gegenüber wem welche Hoheitsbefugnis auf welcher Grundlage und in welchen Grenzen legitim ausüben? So ergab sich etwa schon im 16. Jahrhundert in Württemberg die Frage der Hoheitsbefugnisse des Herzogs gegenüber den Landständen und wurde durch den Tübinger Vertrag von 1514 beantwortet. Vergleichbar stellt sich im 21. Jahrhundert etwa in der Bundesrepublik die Frage nach dem legitimen und kontrollierten Dürfen der Besteuerung und findet ihre erste Antwort im Grundgesetz. Und in der Fragestruktur nicht grundlegend anders erheben sich zu Recht Stimmen, die wissen wollen, ob die Europäische Gemeinschaft die Mitgliedstaaten verpflichten darf, bestimmte Vorgaben für die Mehrwertsteuer zu beachten oder ein Tabakwerbeverbot auch für Lokalzeitungen zu erlassen. Dies aber findet Antwort in den Normen des vertragslegitimierten Primärrechts, das seinerseits die Regeln zur Legitimation und Kontrolle europäischer Hoheitsbefugnisse beinhaltet: Regeln zur Verbandskompetenz, zur Organkompetenz und zur Verfahrensweise; Regeln auch zur gerichtlichen Kontrolle von Maßnahmen der politischen Organe am Maßstab des Primärrechts durch den EuGH, der etwa im ersten TabakwerbeUrteil die betroffene Richtlinie für nichtig erklärte, ${ }^{87}$ im zweiten die Nachfolge-Richtlinie aber bestätigte ${ }^{88}$ - wenn auch mit erstaunlich angreifbarer Begründung.

\section{Zeitloser Topos}

Damit aber zeigt sich, dass die Funktion des Verfassungstopos zeitlos auch im europäischen Kontext ist, soweit es um die Legitimation und Kontrolle von europäischen Hoheitsbefugnissen geht. Semantisch muss ein Text, der Verfassungsinhalte enthält, nicht Verfassung oder Verfassungsvertrag heißen. Das Grundgesetz ist ein Beispiel hierfür. Das (insoweit unverdächtige) Bundesverfassungsgericht nannte schon vor vierzig Jahren den EWG-Vertrag ,gewissermaßen die Verfassung dieser Gemeinschaft“" 89 der EuGH vor 16 Jahren den EG-Vertrag die „Verfassungsurkunde“.$^{90}$ An sie sind alle Mitgliedstaaten gebunden, auch Frankreich und die Niederlande paradoxerweise trotz Ablehnung des Verfas-

86 Vgl. dazu Müller-Graff: Strukturmerkmale des neuen Verfassungsvertrages für Europa, 2004, S. 189f.

87 EuGH, Rs. C-376/98 - Urteil vom 05.10.2000.

88 EuGH, Rs. C-380/03 - Urteil vom 12.12.2006.

89 BVerfGE 22, 293 - EWG-Verordnungen.

90 EuGH, Slg. 1991, I-6102. 
sungsvertrages. Und selbst das Mandat kommt zur Inhaltsbestimmung einer bestimmten Frage des Unionsrechts nicht ohne Rekurs auf den Verfassungstopos aus, wenn es in der Neuformulierung des Art. 6 Abs. 3 EUV formuliert, dass die Grundrechte, wie sie sich aus den gemeinsamen Verfassungsüberlieferungen der Mitgliedstaaten ergeben, allgemeine Grundsätze des Unionsrechts sind. Verlieren etwa diese Eckpunkte zu Legitimation und Kontrolle unionaler Hoheitsbefugnisse diese Verfassungsfunktion durch die Transformation in Grundsätze des Unionsrechts?

So entgeht denn die zeitgerechte Gestaltung des Primärrechts nicht der klassischen verfassungsfunktionalen Aufgabe in einem doppelten Sinne: Erstens der zeitgemäßen Festlegung von Legitimation und Kontrolle hoheitlichen europäischen Handelns; zweitens der Schaffung eines dauerhaften stabilen normativen Rahmens einer spezifischen Ordnung von Frieden, Toleranz, Freiheit, Prosperität, Rechtsgüterschutz, Solidarität und weltweiter Selbstbehauptung für die europäischen Staaten. Hinzuzufügen ist: Gerade in Phasen, in denen nationale Rhetorik aufwallt, bleibt eine derartige Ordnung im Interesse aller Beteiligten und im besonderen Interesse des Landes in der Mitte Europas, das die meisten Nachbarn aufweist.

\section{Ergeb nis}

Abschließend ist festzuhalten: Ein effektives Primärrecht als verbindliche Basis des integrativen Gleichgewichts in Europa im Sinne Werner Links ${ }^{91}$ bietet große Chancen. Es bändigt die gefahrstiftende Unordnung rivalisierender Nationalstaaten. Es ermöglicht gemeinsame Prosperität. Es kann vor allem gerade auch in den Herausforderungen der Globalisierung den Handlungsspielraum der europäischen Nationalstaaten paradoxerweise durch Supranationalität erhalten. Es hat in diesem Sinne die Daueraufgabe, eine tragfähige rechtsnormative Grundordnung für Europa bereitzustellen. Keine Frage: Dieser Verfassungstopos hat Zukunft.

91 Werner Link: Auf dem Weg zu einem neuen Europa. Herausforderungen und Antworten, Baden-Baden 2006. 